\title{
Determining selective surface energy of the native internal rock structure using iGC
}

\author{
M. RÜCKER ${ }^{* 1}$, M. NADERI $^{2}$, D. WILlIAMS ${ }^{1,2}$, A. \\ GEORGIADIS $^{1,3}$, P. F. LUCKHAM ${ }^{1}$
}

${ }^{1}$ Imperial College London, London SW7 2AZ, UK

(*correspondence: m.rucker15@imperial.ac.uk)

${ }^{2}$ Surface Measurement Systems Ltd, Rosemont Rd, Alperton, Wembley HA0 4PE, UK

${ }^{3}$ Shell Global Solutions International B.V., Grasweg 31, 1031

HW Amsterdam, NL

The flow of fluids in porous rocks is subject to geochemical processes involving solid dissolution or precipitation, and solute adsorption or desorption. These are molecular processes, which in turn impact larger-scale multiphase flow properties of the system such as relative permeability. Next to the chemical characteristics of the fluid, these processes also depend on mineralogy, structural characteristics and heterogeneities of the internal rock surface. A challenge in assessing the aforementioned geochemical mechanisms is to upscale these rock properties from representative measurements.

In this work, we investigate the potential for inverse gas chromatography surface energy analyser (iGC SEA) to reduce this gap. With iGC SEA the surface energies and area within the pore space can be determined through the retention time of a selective set of probing gases flowing through $\mathrm{cm}$-sized core samples [1]. We assessed the surface energy and area for two rocks: a chemically homogenous Ketton carbonate ( $99.9 \%$ calcite) and chemically heterogeneous Bandera Brown sandstone (59\% quartz, $46 \%$ plagioclase, $14 \%$ clay) at different humidities. Due to capillary condensation, the humidity could be related to a specific capillary pressure [2]. The variation in humidity and thus capillary pressure provided a basis for assessing mineral-specific surface area exposed to an invading non-wetting fluid.

Previous atomic force microscopy and micro-computed tomography core-flood experiments demonstrated how this relationship relates to the flow behaviour of multiphase flow systems [3]. The workflow encompassing iGC SEA, allows to obtain this relationship on a more representative scale and could be utilized as input for modelling frameworks.

[1] Smith et al. (2014) Langmuir, 30(27), 8029-8035. [2] Rücker et al. (2020) JCIS, 562, 159-169. [3] Sang et al. (2019) WRR, 55, 7212-7228. 